\title{
Beam test results of STS prototype modules for the future accelerator experiments FAIR/CBM and NICA/MPD projects
}

\author{
Petr Kharlamov ${ }^{1}$, Dmitrii Dementev ${ }^{2}$, and Mikhail Shitenkov ${ }^{2}$ \\ ${ }^{1}$ SINP MSU, Department of experimental high energy physics, 119991 Moscow, Russian Federation \\ ${ }^{2}$ LHEP JINR, 141980 Dubna, Moscow region, Russian Federation
}

\begin{abstract}
High-energy heavy-ion collision experiments provide the unique possibility to create and investigate extreme states of stronglyinteracted matter and address the fundamental aspects of QCD. The experimental investigation the QCD phase diagram would be a major breakthrough in our understanding of the properties of nuclear matter. The reconstruction of the charged particles created in the nuclear collisions, including the determination of their momenta, is the central detection task in high-energy heavy-ion experiments. It is taken up by the Silicon Tracking System in CBM@FAIR and by Inner Tracker in MPD@NICA currently under development. These experiments requires very fast and radiation hard detectors, a novel data read-out and analysis concept including free streaming front-end electronics. Thermal and beam tests of prototype detector modules for these tracking systems showed the stability of sensors and readout electronics operation.
\end{abstract}

\section{Double-sided strip detectors in high energy physics}

In Particle Physics experiments particles like electrons, positrons or protons are accelerated to energies in the $\mathrm{GeV}$ to $\mathrm{TeV}$ range and brought to collision, either on a fixed target or head on in colliding beams. [1] The particles or their constituents interact. Determining precisely the properties of the final state particles the interaction process can be studied, e.g. cross sections, angular distributions or asymmetries. Often the main interest is not the primary interaction but the properties of new particles created in the interaction, like heavy quarks, W- or Z-bosons, B-mesons etc. They are unstable and decay almost immediately into secondary (or tertiary) particles which are stable and can be detected. Ideally many different quantities need to be determined in order to allow a complete reconstruction of the interaction or a decay: the particle type, its charge (sign), the momentum vector and energy and for short lived particles the production and decay vertex. However, no detector can measure all these quantities together, detector systems made from sub-detectors each specialized in one or several quantities are needed. In colliding beam experiments the detectors have to cover the interaction region completely (" $4 \pi$ detector"). Hence the sub-detectors are arranged in cylindrical layers around the beam pipe, the forward and backward regions are closed with plug-like detectors. In fixed target 
experiments most of the particles are contained in a narrow cone following the beam direction, hence it is sufficient to have wedge-shaped detectors covering this cone.

One of the main detector components is a tracking detector. In a magnetic field the tracks of charged particles are curved, the curvature is measured by the tracking detector allowing to reconstruct the momentum of the particle. Good momentum resolution requires large detectors, tracking detectors have radii of ( 1-2 ) m. Ideally the tracking detectors and the coil of the magnet should give only minimal disturbance to the trajectory of the charged particles and should not cause interactions of neutral particles.

Thus, the subject of this article is tracking detector modules for the future heavy-ion experiments CBM@FAIR,BM@N and MPD@NICA. The modules consist of doublesided silicon strip sensors and self-triggered electronics. So, this paragraph deals with sensors, and the next paragraph covers electronics.

The operation principle of silicon sensors is simple: They are basically diodes with a pn-junction which is depleted applying a reverse voltage from external electrodes. In such a reverse biased diode only a very small thermally generated current is flowing and a large electric field is built up in the depleted zone. Photons and ionizing particles create electronhole pairs. In the electric field of the depleted diode these charge carriers drift to the electrodes where they induce a signal.

Position information can be obtained by segmenting the electrodes creating many independent diodes. From the known position of the electrode where the signal is induced the position of the ionizing particle can be deduced. In the following sections these aspects will be discussed in more detail.

As electrons and holes in a strip detector are swept by the electric field to opposite sides of the wafer, it is possible to use both types of charge carriers for position measurement by providing charge-collection electrodes on both sides of the wafer (Fig. 1). This doublesided readout brings about the obvious advantage of providing twice the information for the same amount of scattering material.

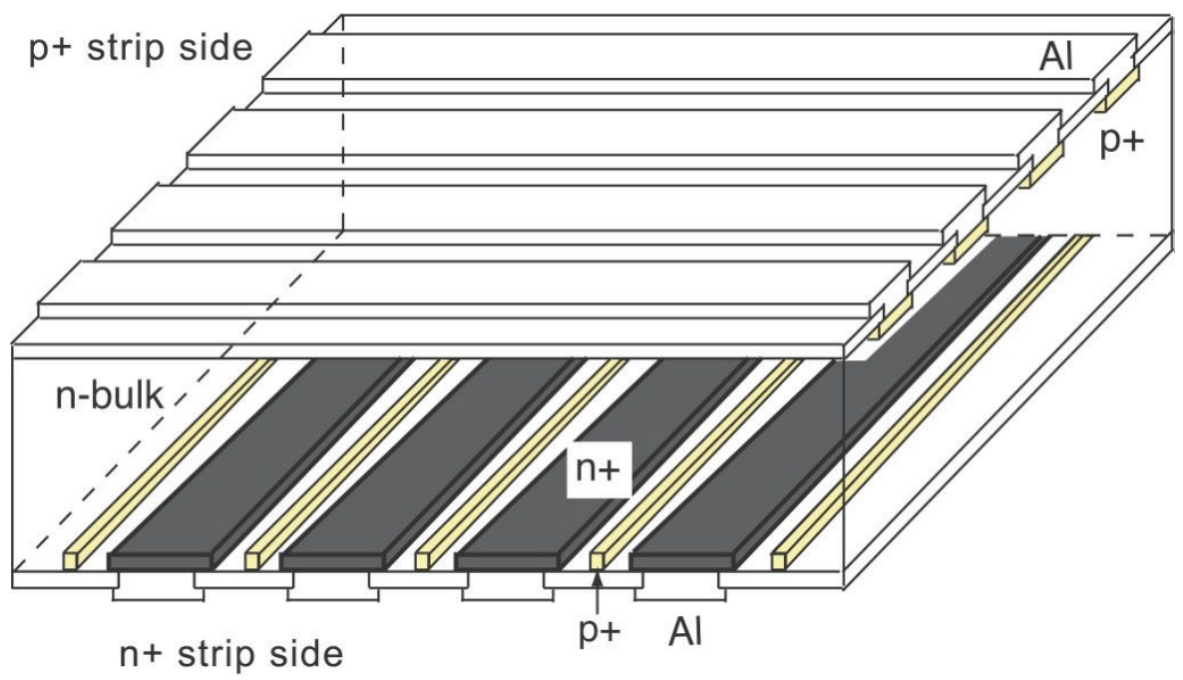

Fig. 1. Sketch of double-sided strip sensor with implanted interleaved $p$-strips between $n^{+}$-charge collection strips

With crossed strips on the two detector faces, projective two-dimensional measurement is obtained from one single detector. For a traversing particle, a spatial point can be reconstructed as both projections are taken from the same initial charge cloud. For absorbed 
radiation such as x-rays, two-dimensional measurement becomes possible. With analog readout it is furthermore possible (to some degree) to correlate signals from the two sides, making use of Landau fluctuations and the exact equality of positive and negative charge created by each ionizing particle. This can be of interest for resolving ambiguities when several particles simultaneously hit the detector.

A problem in producing the double-sided kind of detector is the insulation of each of the strips from the others simultaneously on both sides of the detector. This problem has several solutions. [2] In our sensors the method of disruption of the electron layer by implantation of $\mathrm{p}$ strips between the $\mathrm{n}^{+}$charge-collection strips is used.

\section{Readout electronics}

Modern high energy physics call inevitably for carrying out complex and expensive accelerator experiments. Detectors needed for data acquisition are very large and consist of a big amount of sensors. Information as a whole, in turn, is collected from classified information from the separate parts. In such experiments useful data is obtained by successive reduction of the raw data.

As a rule, only a few interactions occurring in high energy particles collisions are of interest for researchers. As a result, modern detectors are created to quickly examine collision data and, for that reason, to quickly make a decision on importance of this collision. Hardware and software systems making such decisions are called "triggers". They divided into "levels"; each subsequent "level" represents more and more limiting set of criteria, which a signal must answer to pass to the next trigger levels. The signal passing through all trigger levels records as event corresponding to phenomenon. Readout electronics working with such external systems are called triggered.

There is also another type of readout electronics. In such electronics a trigger signal is produced by electronics itself, and so such type of electronics is called self-triggered.

Both types have common problems, for example, signal-to-noise ratio. But there are some specific problems. Triggered electronics is used in collider experiments; bunchcrossing time of them is precisely known, so moment of time of possible events is known. And electronics read nothing all other time. Therefore, reliable trigger system is required. In case of fixed-target experiments (e.g. CBM@FAIR) and/or long bunch of accelerated particles (e.g. MPD@NICA) significant fraction of events will be lost inevitably. In that case self-triggered electronics should be used. It is more difficult to obtain high signal-tonoise for self-triggered electronics; also it usually has bigger energy consumption (and thus heat emission). But in general it is more universal solution for experiment in high energy physics.

In our work self-triggered readout electronics n-XYTER (Neutron-X-Y-Time-Energy Read-out) is used now. It integrates 128 channels with low noise preamplifiers and shapers. [3] Each channel has two different shapers with distinct time constants, one optimized for timing resolution, the other one optimized for energy (pulse height) resolution (Fig. 2). A peak detector connected to the slower shaper allows for the application of a spectroscopic amplitude measurement. An internal time stamp generator provides the temporal reference that may be employed to identify time coincidences of signals on different detector channels and thus correlate their spatial point of origin. For testability and calibration purposes, a charge injector with adjustable pulse height was implemented. The bias settings and various other parameters can be controlled via a standard $\mathrm{I}^{2} \mathrm{C}$-interface. 


\section{n-XYTER Architecture}

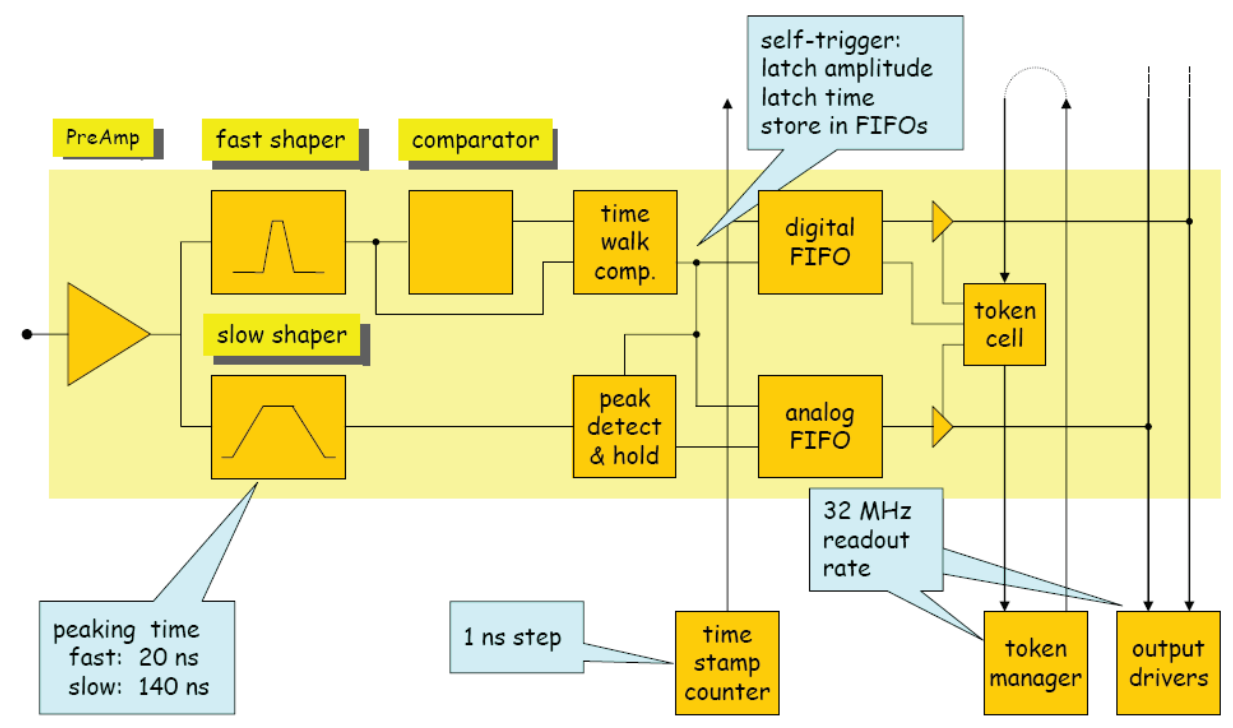

Fig. 2. Flowchart of n-XYTER.

Advantages of n-XYTER: high read rate, works without trigger modules, good energy solution.

Disadvantages of n-XYTER: high heat release (near $6 \mathrm{~W}$ ) [4], big noise, dependence of DC-signal levels on temperature (in n-XYTER 1.0).

Therefore, n-XYTER can be used in spectrometric measurement and for tracking detectors testing, because it can make high-precision measurements of signal height. And because of self-triggered nature, n-XYTER can also be used in fixed-target experiments.

For the CBM@FAIR a new prototype of the STS read-out is currently under development: STS-XYTER2. [5] As well as n-XYTER, the chip uses a low-noise Charge Sensitive Amplifier (CSA) to aggregate the charge generated in the detector active volume and to convert it into the voltage step. The signal divides into fast and slow paths too. The fast path is optimized for timestamping of events and uses a single-stage shaper with $30 \mathrm{~ns}$ shaping time, a discriminator and a 14-bit timestamp latch working with Gray-encoded timestamp counter. The slow path consists of a CR-(RC) ${ }^{3}$ shaper working with a 5-bit flash $\mathrm{ADC}$ and a digital peak detector. It is optimized for low-noise charge measurement. The shapers' design is very similar to the implementation in the previous prototype chip, STSXYTER, and n-XYTER.

\section{Tracking systems of CBM@FAIR, BM@N and MPD@NICA experiments}

High-energy heavy-ion collision experiments provide the unique possibility to create and investigate extreme states of strongly-interacted matter and address the fundamental aspects of QCD such as equation-of-state, structure and properties of nuclear matter and modification of hadrons in nuclear medium. At large baryon chemical potentials QCD phase diagram features structures such as a critical point and transitions between various phases such as hadronic phase, quarkonic phase, quark-gluon plasma and even colour 
superconductors. The experimental discovery of these landmarks of the QCD phase diagram would be a major breakthrough in our understanding of the properties of nuclear matter.

However, due to luminosity limitations modern experiments suffer from statistics for rare diagnostic probes. In order to overcome this limitation, the new projects are under development at the Facility for Antiproton and Ion Research (FAIR) in Darmstadt and at the Nuclotron-based Ion Collider fAcility in Dubna. And so, the detectors of these experiments are designed to measure rare diagnostic probes such as multy-strange hyperons, charmed particles and vector mesons decaying into lepton pair with unprecedented precision and statistics. Most of these particles will be studied for the first time in the FAIR and NICA energy range. In order to achieve the required precision, the measurements will be performed at reaction rates up to $10 \mathrm{MHz}$. This requires very fast and radiation hard detectors, a novel data read-out and analysis concept including free streaming front-end electronics, and a high performance computing cluster for online event selection.

The reconstruction of the charged particles created in the nuclear collisions, including the determination of their momenta, is the central detection task in these experiments. In CBM experiment it is taken up by the Silicon Tracking System, an array of eight tracking stations based on silicon micro-strip sensors (Fig. 3) currently under development. [6] In the innermost parts of the first two stations, where particle occupancy is expected to reach $5 \%, 2 \mathrm{~cm}$ high sensors have been chosen: such granularity allows minimizing the number of ghost hits. However, to reduce material budget and to minimize the number of read-out chips, it is possible to use at the outer parts of the stations, where occupancies are less than $5 \%$, up to two $6 \mathrm{~cm}$ sensors daisy-chained together, creating effective strip lengths up to about $12 \mathrm{~cm}$. Every sensor has a fixed horizontal width of $6 \mathrm{~cm}$ (Fig. 4).

Because of identity of BM@N experiment in essence (it is also used for testing of equipment for CBM) STS is the same as that of CBM but it has less stations. [7] Both of CBM and BM@N are fixed-target experiments and have single-arm spectrometer detectors. MPD@NICA is a collider experiment, and so it has cylinder of many layers detector.

One of the main detector systems of MPD is the Inner Tracker. It consists of four concentric cylinders of silicon strip detectors (Fig. 5). [8] It consists of a silicon barrel and a system of silicon discs. The mechanical support and cooling elements of both the cylinders and the discs are manufactured out of carbon fiber to minimize the material budget of the setup and hence to diminish the impact of the Coulomb multi-scattering on track resolution. The double sided SSDs of the four cylinder-like sensitive layers of the barrel are mounted on the 3-D light Carbon Fiber (CF) space frames similar to those developed for the inner tracker of ALICE (CERN) and STAR (BNL). The stereo angle between the strips on the opposite sides of the SSDs is $\alpha=7.5^{\circ}$. The optimization of the value of stereo angle is important for minimizing the number of ambiguities in track reconstruction. From typical strip pitch of $\mathrm{h}=58 \mu \mathrm{m}$ yields a space resolution of a track close to $\sigma_{\phi}=\mathrm{h} /(\sqrt{12})=17 \mu \mathrm{m}$ in $(r, \phi)$ direction, and $\sigma_{\mathrm{z}}=\mathrm{h} /(\sqrt{12} * \alpha)=128 \mu \mathrm{m}$ in $\mathrm{Z}$ the direction along the beam line. To eliminate the effect of insensitive areas at the edges of the sensors, the ladders are positioned with a small overlap. 


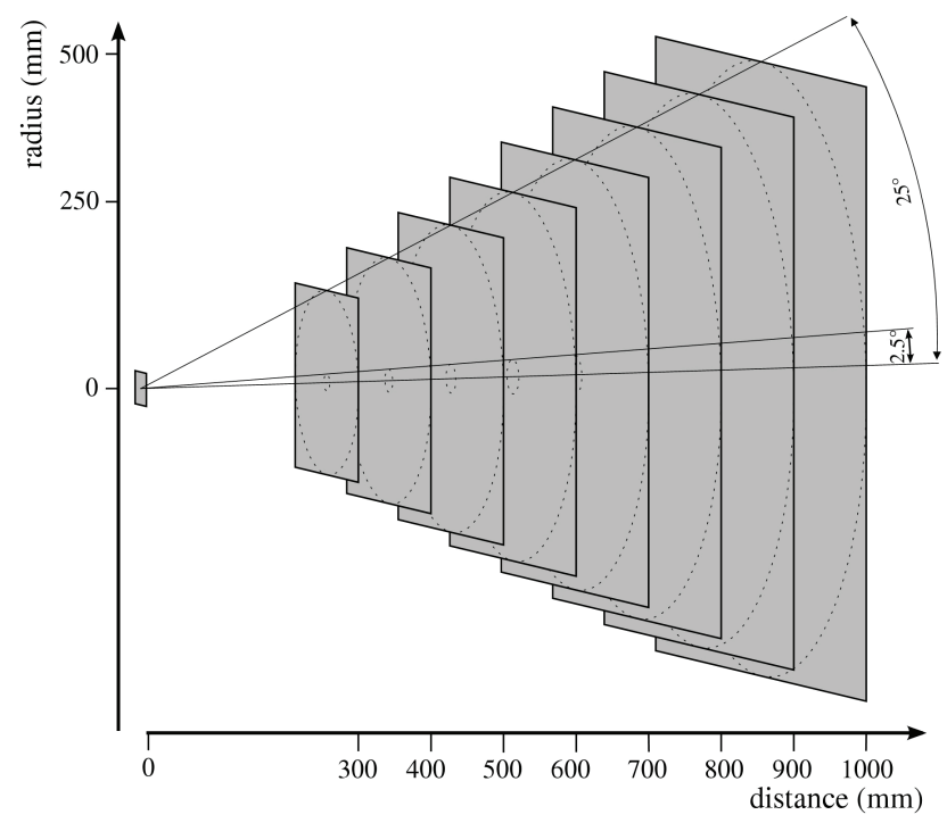

Fig. 3. Concept of STS tracking stations covering the polar angles $2.5^{\circ}<\Theta<25^{\circ}$.

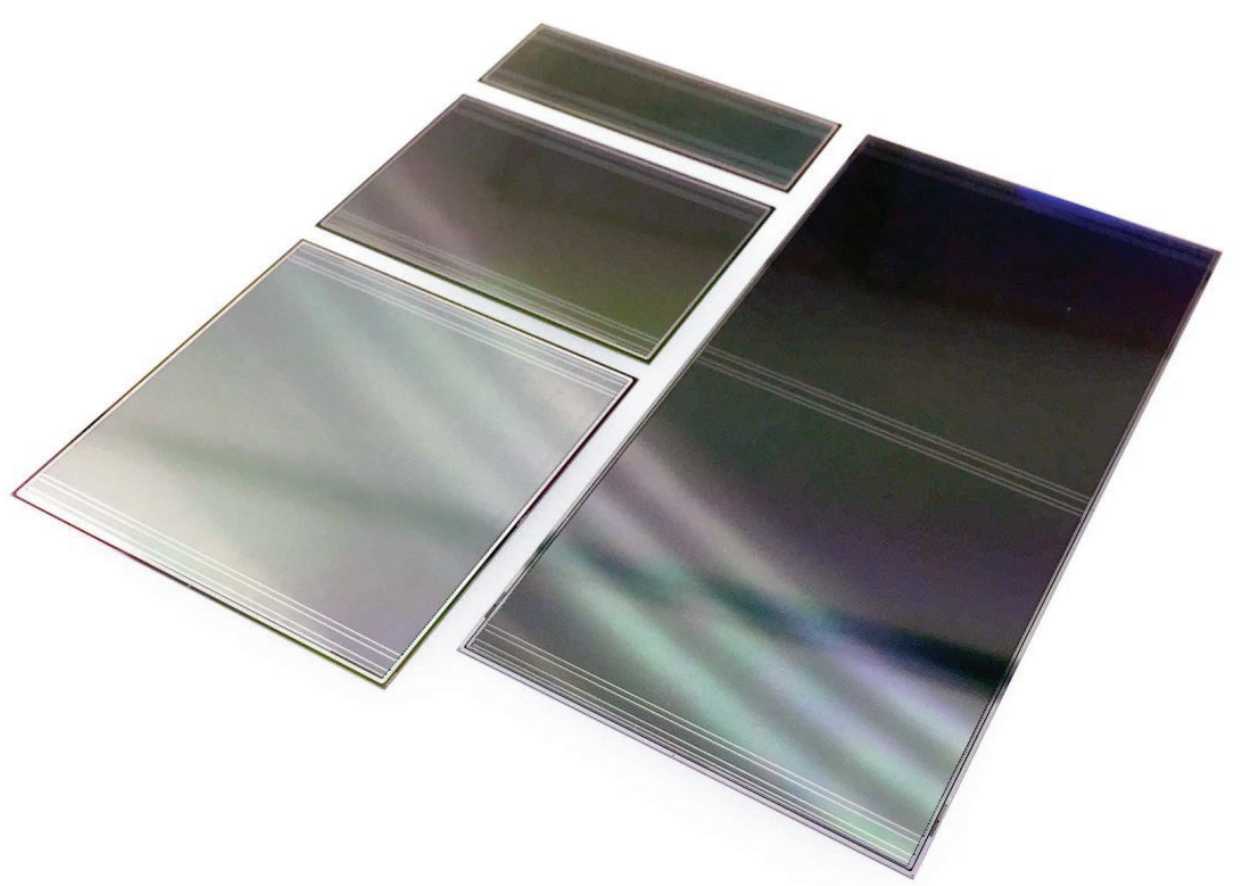

Fig. 4. Sensors $6 \times 2,6 \times 4,6 \times 6$ and $6 \times 12 \mathrm{~cm}^{2}$. 


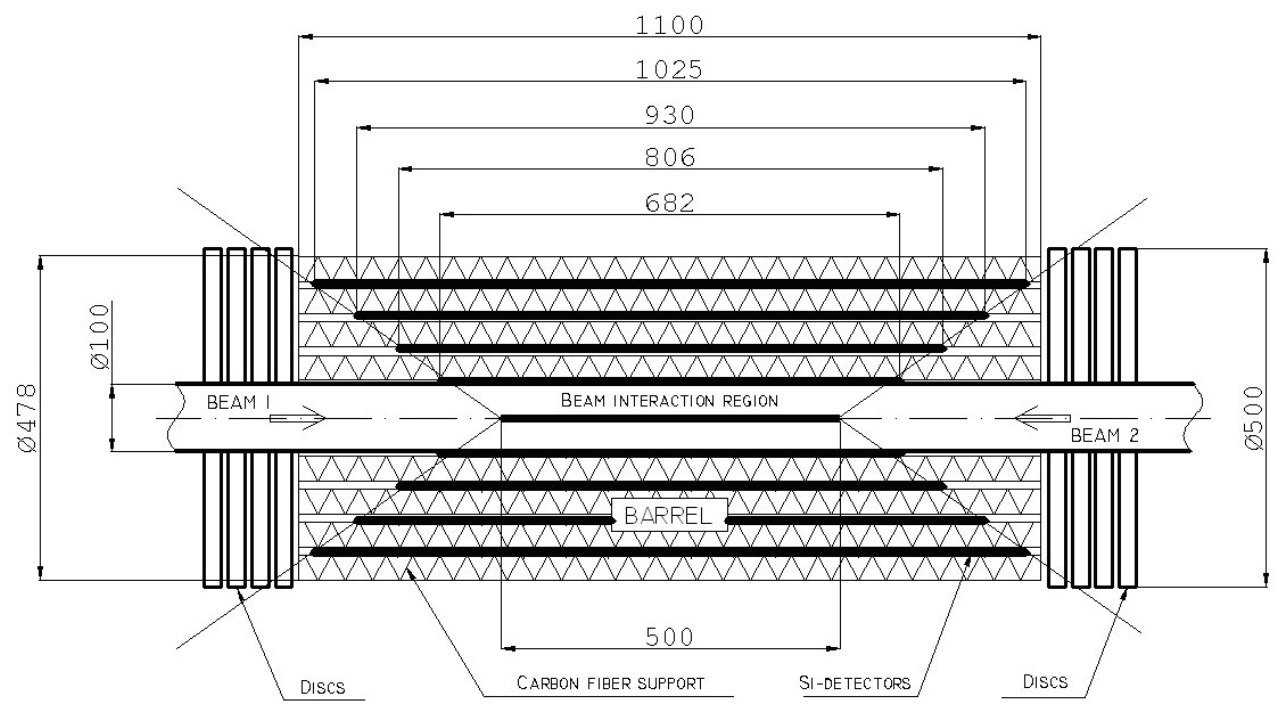

Total number of detectors 806

Fig. 5. Concept of Inner Tracker stations.

\section{Tests of tracking modules}

Sensors and electronics tests were carried out using experimental setup located in NUCLOTRON NIS-GIBS zone. There were two variants of setup with $6 \times 6 \mathrm{~cm}^{2}$ sensor and $14.9 \times 14.9 \mathrm{~mm}^{2}$ baby-sensor (Fig. 6).

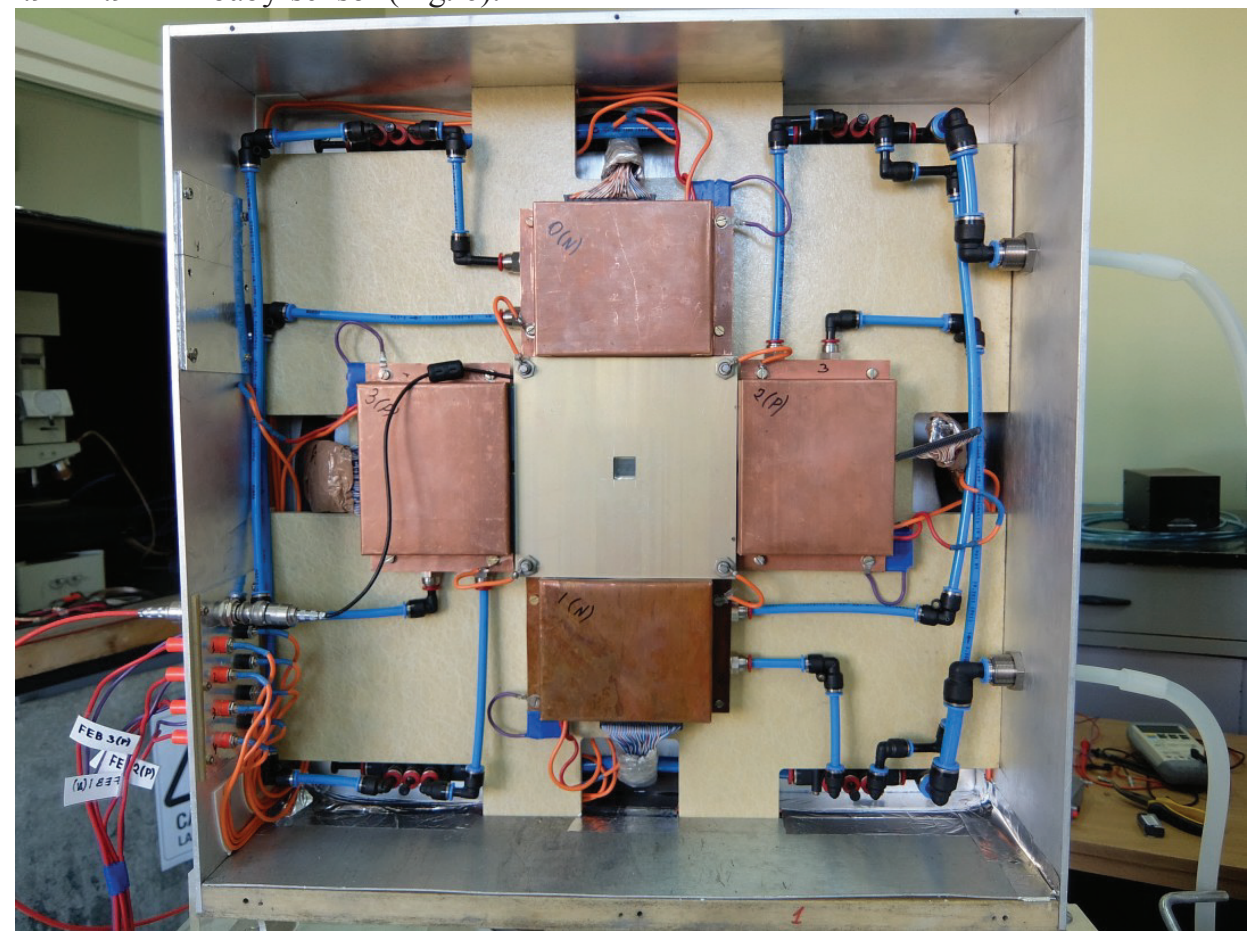

Fig. 6. Test station with baby-sensor (in center) and 4 Front-end boards. 
Tests were carried out during two accelerating sessions of NUCLOTRON: session №53 had deuterons of kinetic energy $4.5 \mathrm{GeV}$ with intensity $\mathrm{I}=2 * 10^{5} \mathrm{c}^{-1}$ and session №54 had deuterons of kinetic energy $2.94 \mathrm{AGeV}$ and the same intensity.

Signals pickup from two detector modules was performed; the signals characteristics meet expectations. On the left side of spectrum noise of Gaussian form prevails; the form of main signal is the Landau distribution (Fig. 7). The signals were taken for the different angles between sensor and beam in order to determine dependence of average cluster size of sensor on that angle. As expected, cluster-1 event count decreases with increasing of the angle and merges into the noise. For the angle of $19^{\circ}$ the signal cannot be distinguished from the noise (Fig. 8).

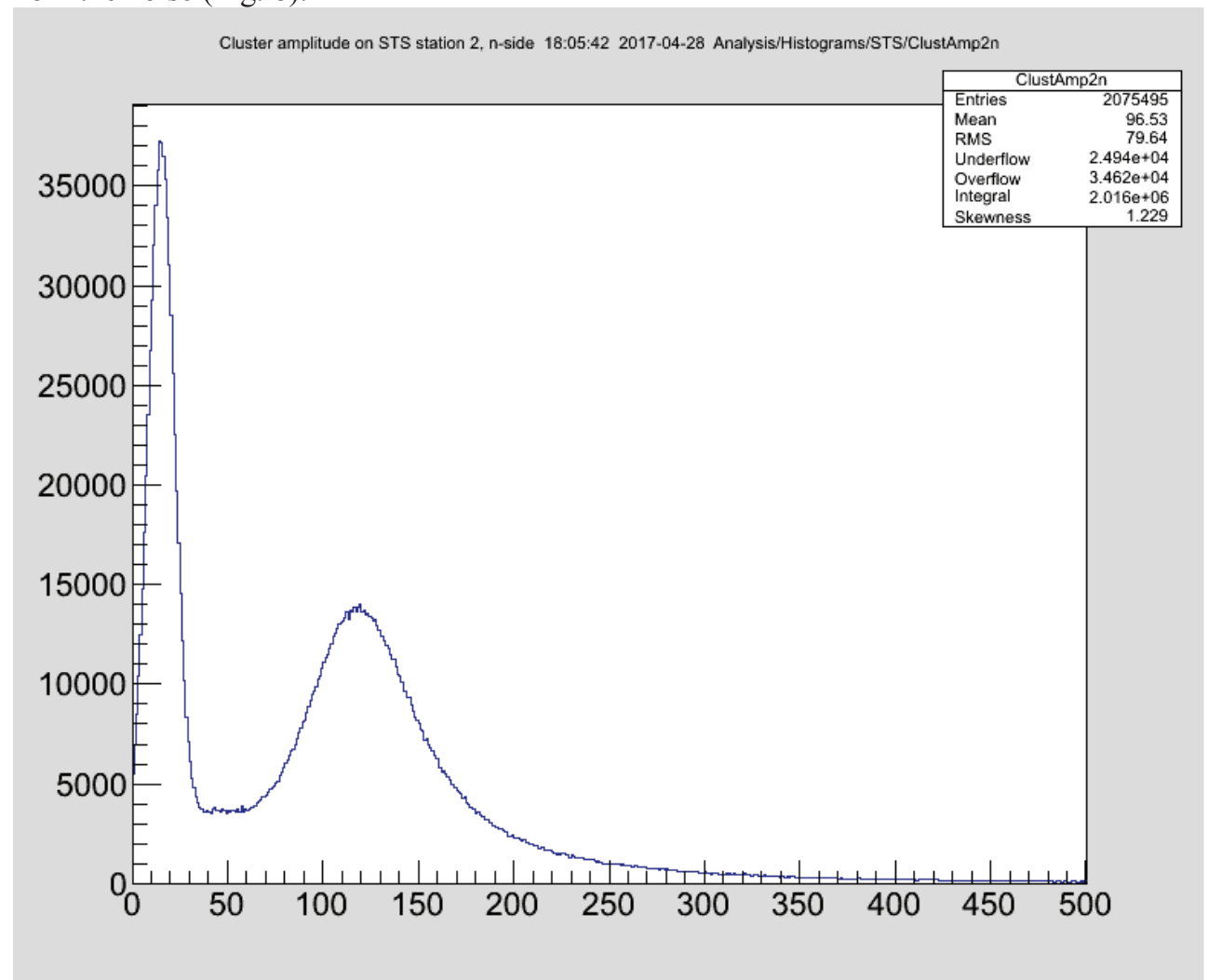

Fig. 7. Sensor $6 \times 6$ signal (n-side).

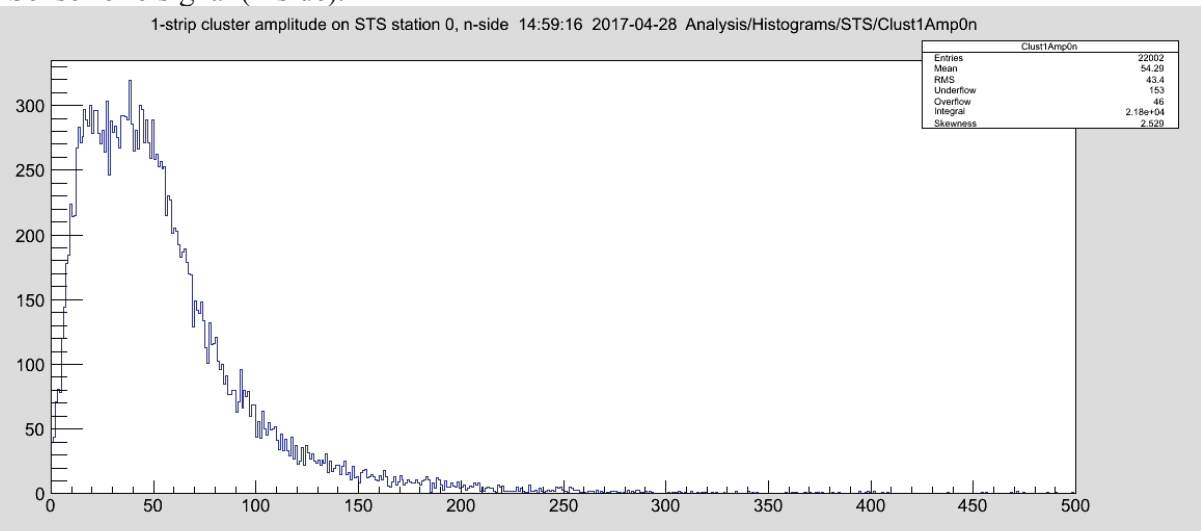

Fig. 8. Cluster-1 event count for baby-sensor (by the angle of $19^{\circ}$ ). 
Average cluster sizes for the angles of $0^{\circ}, 9^{\circ}, 19^{\circ}$ and $27^{\circ}$ were calculated, and dependence on the angle was plotted (Fig. 9). The dependence can be approximated by a quadratic polynomial good enough. Experimental dependence can be compared to that from modeling; experimental cluster sizes are significantly less. This is due to fact that modeling deal with ideal sensors and electronics. In reality on the assumption of big angles energy of ionization divided into about 5 strips, and the signals from the leftmost and rightmost strips swamped by noise and cannot be counted. It leads to real cluster size be considerably decreased.

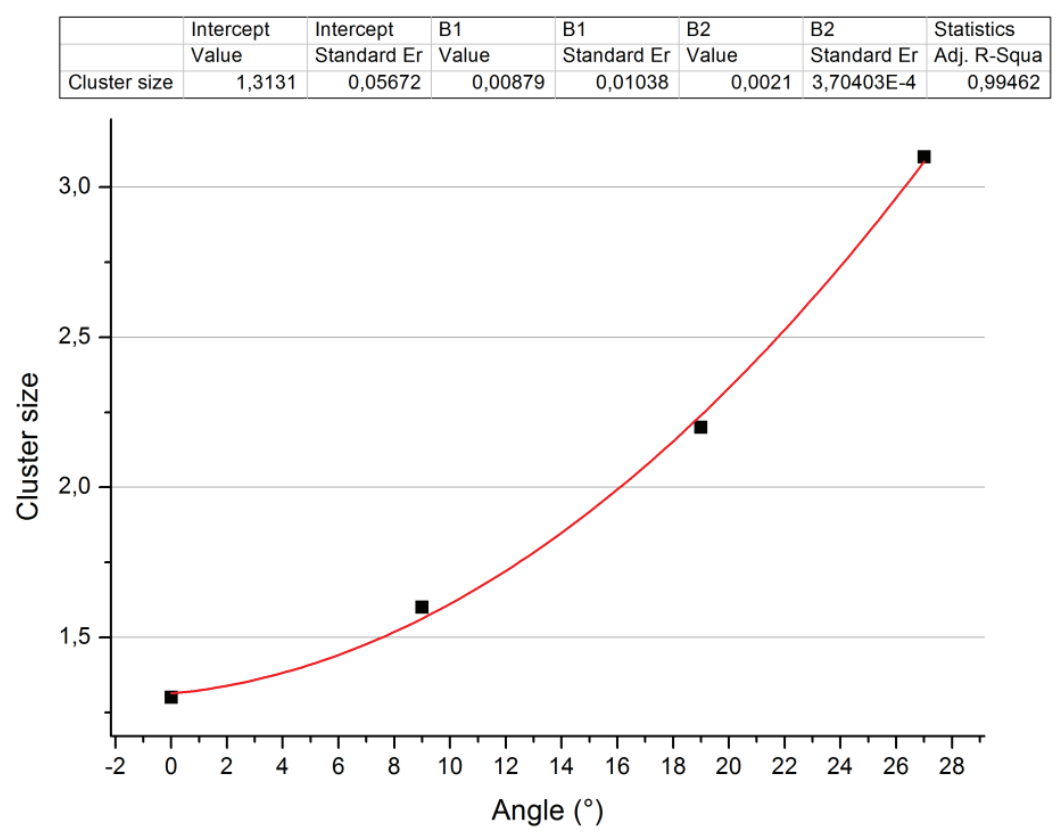

Fig. 9. Cluster size dependence.

Also, it should be noted that temperature tests of detector modules were carried out. [9] After heating, defects were not found. Modules work as expected.

\section{Conclusions}

All systems of the experimental setup work in normal mode. Used techniques of detector modules manufacturing and assembling are suit well for the future experiments. It will be necessary to test detector modules with new and more appropriate readout electronics (STS-XYTER) and to solve self-triggered electronics problems, such as signalto-noise ratio and clocks synchronization of different modules and real time of events. Also it is necessary to check algorithms of track reconstruction. 


\section{References}

1. H.-G. Moser, Progress in Particle and Nuclear, Physics Silicon detector systems in high energy physics, p. 186-237, (2009)

2. G. Lutz, Semiconductor Radiation Detectors, (2007)

3. A.S. Brogna, S. Buzzetti, C.J. Schmidt et al., The n-XYTER Reference Manual, (2009)

4. P. Kharlamov, Testing of self-triggered readout electronics for double-sided microstrip silicon sensors based on n-XYTER ASIC, Summer students program at JINR (2015)

5. Kasinski K., Kleczek R. and Szczygiel R., Front-end readout electronics considerations for Silicon Tracking System and Muon Chamber, Journal of Instrumentation, V. 11. (2016)

6. J.M. Heuser, W.F.J. Müller, V. Pugatch et al., Technical Design Report for the CBM : Silicon Tracking System (STS), (2013).

7. T.O.Ablyazimov, P.G.Akishin, V.P.Akishina et al., Conceptual Design ReportBM@N - Baryonic Matter at Nuclotron, (2013)

8. A.N. Sissakian, A.S. Sorin, V.D. Kekelidze et al., The MultiPurpose Detector - MPD to study Heavy Ion Collisions at NICA (Conceptual Design Report), (2011)

9. Dementyev D., Shitenkov M., and Kharlamov P., Thermal tests of CBM STS prototype modules on $C F$-frames, Compressed Baryonic Matter Experiment at FAIR - Progress Report 2016, (2017) 